\title{
Optimum Detection of Ultrasonic Echoes Applied to the Analysis of the First Layer of a Restored Dome
}

\author{
Luis Vergara, Ignacio Bosch, Jorge Gosálbez, and Addisson Salazar \\ Departamento de Comunicaciones, Universidad Politécnica de Valencia, 46022 Valencia, Spain
}

Received 26 February 2007; Accepted 19 June 2007

Recommended by William Allan Sandham

Optimum detection is applied to ultrasonic signals corrupted with significant levels of grain noise. The aim is to enhance the echoes produced by the interface between the first and second layers of a dome to obtain interface traces in echo pulse B-scan mode. This is useful information for the restorer before restoration of the dome paintings. Three optimum detectors are considered: matched filter, signal gating, and prewhitened signal gating. Assumed models and practical limitations of the three optimum detectors are considered. The results obtained in the dome analysis show that prewhitened signal gating outperforms the other two optimum detectors.

Copyright ( 92007 Luis Vergara et al. This is an open access article distributed under the Creative Commons Attribution License, which permits unrestricted use, distribution, and reproduction in any medium, provided the original work is properly cited.

\section{INTRODUCTION}

In [1], the authors have considered the ultrasonic echo pulse technique to help in the analysis of a dome. The first four layers of the dome were, respectively, mortar $(0.3 \mathrm{~cm})$, plaster $(1.2 \mathrm{~cm})$, mortar $(1.5 \mathrm{~cm})$, and bricks. The work presented in paper [1] was devoted to the problem of determining the state of adhesion of the interface between the third and fourth layers. The depth of such an interface $(3 \mathrm{~cm})$ and the type of materials (mortar-bricks) allowed working with a transducer of $1 \mathrm{MHz}$ so that grain noise, due to reflections from the micro-grains of the involved materials, is not present at all. No sophisticated signal processing techniques were required in [1]. Actually, the first two interfaces were not detected at $1 \mathrm{MHz}$ of operating frequency, and the only echoes were obtained from the mortar-bricks interface.

The problem considered in this paper is outlining the first interface which is only at a depth of $0.3 \mathrm{~cm}$. This implies the need for increasing spatial resolution and we require transducers with higher operating frequencies to reduce the wavelength. The consequence will be the apparition of significant amounts of grain noise, thus leading to the need of using the statistical signal processing techniques presented in this paper.

The first layer of the dome is a $0.3 \mathrm{~cm}$ stratum of mortar, and the second one consists of a $1.2 \mathrm{~cm}$ stratum of plaster. The objective is to trace the interface between the first and second layers to provide valuable information to the restorers. Information about the state of conservation of the first layer is especially important, as this is usually painted over. Essentially, we want to determine if the layer of mortar is present or not in a given location of the dome under analysis. This is needed by the restorer before to proceed with the restoration to paint. If the layer of mortar is not present in a given location, it is necessary to add some mortar and then painting over it. Mortar layer could not be present because of deterioration due to the pass of time. It is not always easy to visually determine the presence or absence of the layer of mortar, hence ultrasonic information may be valuable for the restorer. Note that the technique is not intended to detect variation in mortar thickness, although, in principle, it could be possible to obtain such information if more than one successive echoes of the mortar-plaster interface could be traced and the ultrasound speed of propagation in mortar could be assumed or estimated. The minimal detectable mortar thickness will depend on the pulse time duration.

We thus carried out a nondestructive ultrasonic analysis using the echo pulse inspection mode: an ultrasonic pulse is sent into the first layer of the mortar, expecting reception of the echo from the mortar-plaster interface. We successively locate the sensor along a vertical linear array of locations. At every location we collect an A-scan (a record of the signal echoed by the material). Finally, aligning the A-scans one under the other, we built a B-scan where, hopefully, the interface 


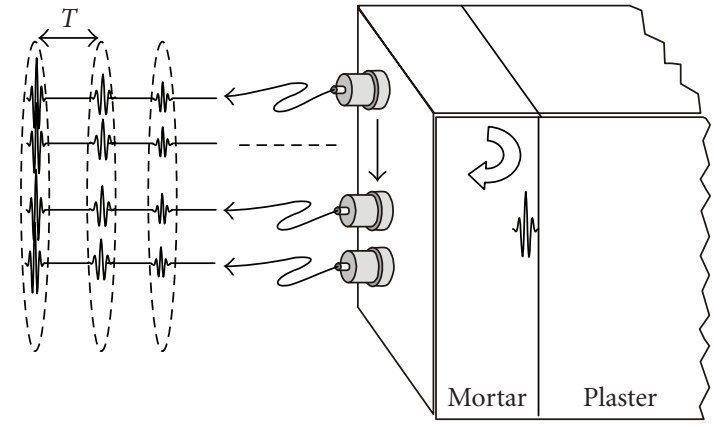

FIGURE 1: Schematic representation of the first layer interface.

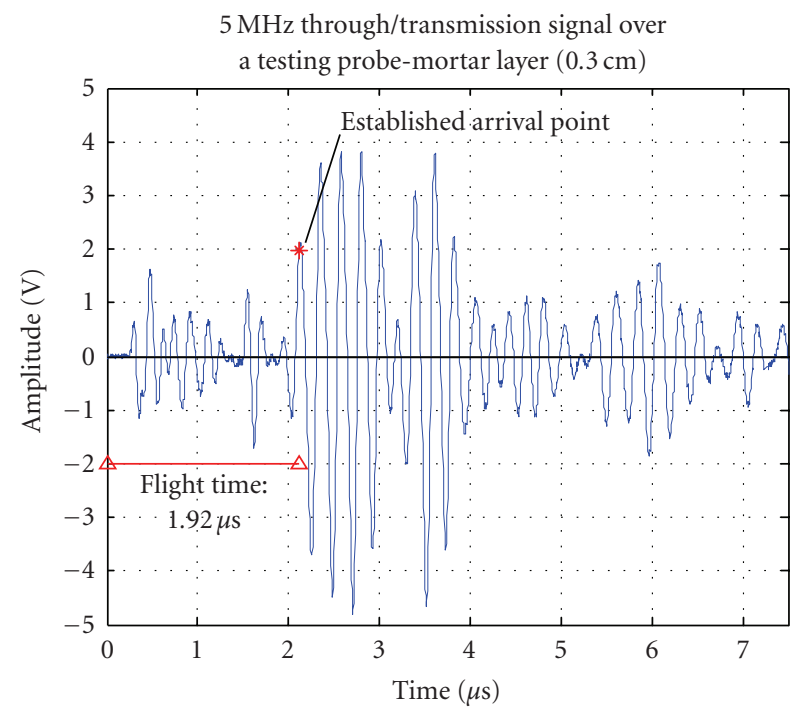

Figure 2: A-scan corresponding to the estimation of the delay in $0.3 \mathrm{~cm}$ mortar thickness.

would be outlined (see the scheme in Figure 1, where possible multiple reflections in the interface have been considered). Gel contact was used for coupling the sensor to the wall.

With the aim of selecting the most appropriate transducer, some experiments were made with 1 and $2 \mathrm{MHz}$, but the spatial resolution was too low. We also tested a $10 \mathrm{MHz}$ transducer, but attenuation was too high to allow reception of the interface echoes. Finally, a $5 \mathrm{MHz}$ transducer was selected to give an adequate balance between resolution and the capacity to penetrate into the mortar. It should be noted that mortar is a material composed of sand and cement paste. Two essential parts of its microstructure are air pores (sizes may vary from $10^{-10}$ to $\left.10^{-4} \mathrm{~m}\right)$ and sand grains $\left(10^{-4}\right.$ to $\left.10^{-3} \mathrm{~m}\right)$. On the other hand, we have estimated the speed of propagation in this type of mortar by using transmission mode in a cylindrical section which was built specifically for this goal. A value $c=1562,5$ (the received signal and the corresponding delay are shown in Figure 2) was obtained so that the wavelength corresponding to $5 \mathrm{MHz}$, $\lambda=c / f=1562.5 / 5 \cdot 10^{6}=0,312 \cdot 10^{-3} \mathrm{~m}$, is of the order of the sand grains diameter. That means that significant

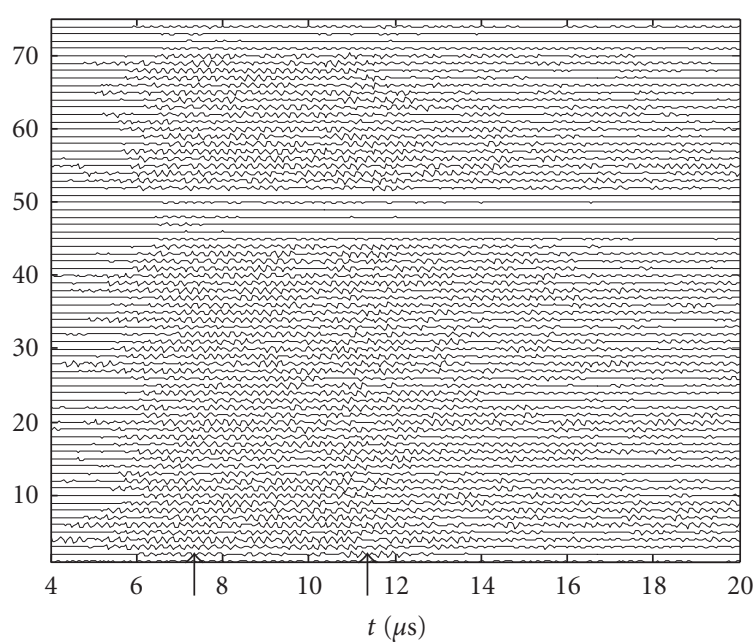

Figure 3: A portion of the original B-scan; the arrows indicate the delays where the trace of the mortar layer interface should be outlined.

amounts of grain noise should be expected, probably hiding the echoes from the first and second layer interface.

The expectation was certainly true, as one can verify by observing Figure 3 where we represent a portion of the original B-scan and two arrows indicating delays where the interface should be outlined (the details of the experiment are described in Section 4). Hence, signal processing is necessary in this case to enhance the presence of the interface echoes (if possible). This problem may be approached in an optimum manner in different ways. The most obvious way is that of maximizing signal-to-noise ratio (SNR) at the output of the processor, but it is also possible to think about maximization of the probability of detection of the interface echoes in a grain noise background. This latter approach is the one selected here, although, for an appropriate definition of SNR maximization, both approaches are equivalent, as we mention in Section 2.

The paper is set out as follows. First, in the next section we define the problem from an optimum detection perspective. Then in Section 3 we derive the different solutions corresponding to different assumptions about the implicit models. Finally, in Section 4 we apply the optimum detection algorithms to the problem in hand. Some conclusions end the paper.

\section{OPTIMUM DETECTION APPROACH}

We wish to detect the presence of a possible ultrasonic echo pulse $p(n)$ in a segment of the recorded and sampled ultrasonic signal $r(n)$. Therefore, we have two possible hypotheses

$$
\begin{array}{ll}
H_{1} & r(n)=p(n)+g(n), \quad n=n_{s}, \ldots, n_{s}+N-1, \\
H_{2} & r(n)=g(n),
\end{array}
$$

where $n_{s}, n_{s}+N-1$ are, respectively, the starting and the final sample numbers delimiting the segment (i.e., $N$ is the segment length), and $g(n)$ corresponds to the grain noise samples under hypothesis $i$. 
Detecting the presence of $p(n)$ implies some processing $f[\cdot]$ on the segment

$$
z\left(n_{s}\right)=f[\mathbf{r}], \quad \mathbf{r}=\left[r\left(n_{s}\right) \cdots r\left(n_{s}+N-1\right)\right]^{T},
$$

and comparison with a threshold

$$
\begin{array}{ll}
\text { if }\left|z\left(n_{s}\right)\right|>t & \text { decide } H_{1}, \\
\text { if }\left|z\left(n_{s}\right)\right|<t & \text { decide } H_{2} .
\end{array}
$$

If we move the value $n_{s}$ along the recorded signal, we may obtain a nonbinary output signal in the form

$$
r_{\text {out }}\left(n_{s}\right)= \begin{cases}r\left(n_{s}\right) & \text { if } H_{1} \text { is decided } \\ 0 & \text { if } H_{2} \text { is decided }\end{cases}
$$

which is the output sequence after processing the input sequence $r\left(n_{s}\right)$.

Optimum design of $f[\cdot]$ can be made by maximizing the signal-to-noise ratio enhancement (SNRE) factor

$$
\begin{aligned}
\mathrm{SNRE} & =\frac{\mathrm{SNR}_{\text {out }}}{\mathrm{SNR}_{\text {in }}}, \\
\mathrm{SNR}_{\text {out }} & =\frac{E\left[r_{\text {out }}\left(n_{s}\right) / H_{1}\right]}{\sqrt{E\left[\left|r_{\text {out }}\left(n_{s}\right)\right|^{2} / H_{2}\right]}}, \\
\mathrm{SNR}_{\text {in }} & =\frac{E\left[r\left(n_{s}\right) / H_{1}\right]}{\sqrt{E\left[\left|r\left(n_{s}\right)\right|^{2} / H_{2}\right]}},
\end{aligned}
$$

where $E[\cdot]$ means statistical expectation. It can be easily shown (see, e.g., [2, page 111]) that

$$
\mathrm{SNRE}=\frac{\mathrm{PD}}{\mathrm{PFA}^{0.5}},
$$

where PD and PFA are, respectively, the probability of detection and the probability of false alarm corresponding to the detection problem defined in (1)-(3). Hence, maximizing PD for a given PFA (Neyman-Pearson optimum detector) in (1)-(3) implies maximization of SNRE for all the possible gating post-processors of the type (4). Thus, optimum design of $f[\cdot]$ implies solving an optimum detection problem, and this will be the approach adopted in this paper.

\section{OPTIMUM DETECTORS}

Let us start from the detection problem defined in (1). We will consider in the following the Neyman-Pearson criterion for the design of the optimum detectors. Note that maximizing PD for a given PFA is more suitable for ultrasonic pulse detection, as it is in other related areas like radar or sonar, where the "a priori" probability of $H_{1}$ is much smaller than the "a priori" probability of $\mathrm{H}_{2}$. Let us consider the different models, their corresponding optimum solutions, and the practical limitations.
Model 1

We assume the following:

(i) perfect knowledge of vector $\mathbf{s}$ defined by $\mathbf{p}=a \cdot \mathbf{s}$; $\mathbf{s}^{T} \mathbf{s}=1, \mathbf{p}=\left[p\left(n_{s}\right) \cdots p\left(n_{s}+N-1\right)\right]^{T}$.

(ii) $\{g(n)\}$ is locally stationary Gaussian inside every interval $\left[n_{s}, n_{s}+N-1\right]$ having the power spectrum $S_{g}(\omega)$.

The optimum solution is the well-known matched filter detector ([3] and the appendix)

$$
z\left(n_{s}\right)=f(\mathbf{r})=\mathbf{r}^{T} \mathbf{C}_{g}^{-1} \mathbf{s},
$$

where $\mathbf{C}_{g}=E\left[\mathbf{g g}^{T}\right]$ is the grain noise local covariance matrix.

Note that the value $a$ in model 1 is not required. It will depend on the object reflectivity and on the attenuation of the pulse in the go and return path through the first layer. Besides, it will be affected by the surface and by the pressure on the transducer in the manual measurement. In detection theory, the test is said to be uniformly most powerful (UMP) in the unknown parameter $a$.

On the other hand, the spectrum of the grain noise $S_{g}(\omega)$ (and so the covariance matrix $\mathbf{C}_{g}$ ) can be estimated to some extent if some training material samples, resembling the actual operating materials under test, were available. It could be estimated also from sample records measured on the specimen under test if they are mostly composed by grain noise.

Finally, the vector $\mathbf{s}$, which represents the form of the pulse to be detected, depends on the pulse arriving at the possible reflector, which, due to the propagation effects, is a distorted version of the actual pulse sent into the material. It also depends on the reflector itself. Some approximation to $s$ could be obtained by "offline" estimation of the pulse waveform using a material with good propagation properties for ultrasound at the corresponding operating frequency. But good knowledge of $\mathbf{s}$ cannot be assumed in general. Let us consider some simple forms to overcome the need for estimating $\mathbf{s}$.

\section{Model 2}

Same as Model 1 regarding the grain noise model, but we assume, with respect to the pulse, that: $\mathbf{C}_{g}^{-1} \mathbf{s}=\mathbf{k}, \mathbf{k}=$ $[k 0 \cdots 0]^{T}$.

From (7), the optimum solution is a simple gating of the original signal

$$
z\left(n_{s}\right)=f(\mathbf{r})=\mathbf{r}^{T} \mathbf{k}=k \cdot r\left(n_{s}\right) .
$$

The above assumption is a simple form to overcome the need for estimating $\mathbf{s}$ (note that knowledge of $k$ is not required as this factor can be absorbed by the threshold $t$ in (3)). Unfortunately, there are no arguments justifying that the assumption, which makes optimum the gating detector, will be verified in general. Hence, one should not expect good results using a simple gating detector except for cases of high signalto-noise ratio (but in these cases all detectors work fine). 


\section{Model 3}

Same as Model 1 regarding the grain noise model, but we assume, with respect to the pulse, that $\mathbf{C}_{g}^{-1 / 2} \mathbf{s}=\mathbf{k}, \mathbf{k}=$ $\left[\begin{array}{llll}k & 0 & \cdots & 0\end{array}\right]^{T}$.

From (7), the optimum solution is a gating of the signal pre-whitened by matrix $\mathbf{C}_{g}^{-1 / 2}$,

$$
z\left(n_{s}\right)=f(\mathbf{r})=\mathbf{r}^{T} \mathbf{C}_{g}^{-1 / 2} \mathbf{C}_{g}^{-1 / 2} \mathbf{s}=\mathbf{r}_{w}^{T} \mathbf{k}=k \cdot r_{w}\left(n_{s}\right),
$$

where $\mathbf{r}_{w}=\mathbf{C}_{g}^{-1 / 2} \mathbf{r}$.

The above assumption is again a simple form to overcome the need for estimating s. In this case, some justification may be found about the general verification of the assumed pulse model. It is clear that matrix $\mathbf{C}_{g}^{-1 / 2}$ implements a linear transformation that "whitens" the grain noise component in $\mathbf{r}(1)$ :

$$
\begin{aligned}
E\left[\mathbf{g}_{w} \mathbf{g}_{w}^{T}\right] & =\mathbf{C}_{g}^{-1 / 2} E\left[\mathbf{g g}^{T}\right] \mathbf{C}_{g}^{-1 / 2} \\
& =\mathbf{C}_{g}^{-1 / 2} \mathbf{C}_{g} \mathbf{C}_{g}^{-1 / 2}=\mathbf{I} \Longleftrightarrow S_{g w}(\omega)=1 .
\end{aligned}
$$

But the assumption with respect to the pulse implies that $\mathrm{C}_{g}^{-1 / 2}$ also "whitens" the spectrum of the pulse (it changes $\mathbf{s}$ to a delta vector $\mathbf{k}$ ). This suggests that this assumption is equivalent to consider that grain noise has a generative model consisting in white noise filtered by a linear filter having impulse response s. This is a simple but a reasonable model if we take into account that grain noise could be considered the result of the superposition of many echoes coming from the material grains, that is, the result of convolving the material reflectivity with the ultrasonic pulse sent into it [4]. As far as this linear generative model of grain noise could be a good approximation of the actual behavior of the material, one should expect good results by using the optimum detector (9).

In the following, we will consider the three optimum detectors for the problem in hand.

\section{ANALYSIS OF THE FIRST LAYER OF A RESTORED DOME}

The research of this work is done under the framework of a collaboration between our Signal Processing Group and the Institute for Cultural Heritage Conservation of the Polytechnic University of Valencia. A final goal was to develop a versatile prototype for ultrasonic nondestructive testing which could be applied to different problems relative to restoration of domes or walls in historical buildings. Versatility was achieved by allowing the use of different sensors and by developing different signal processing modules, both things adapted to every particular problem. That is, for example, in [1] we described a different problem which was worked with essentially the same equipment, but using a different sensor and different (simpler) signal processing algorithms. Of course some parameters to set up the equipment must be also selected for every problem (amplifier gain, analog filter, sampling frequency, etc.). On the other hand, a requirement is that this equipment could be used by people with no special skills in ultrasonics or in signal processing: the user interface must be simple and the calibration must be essentially

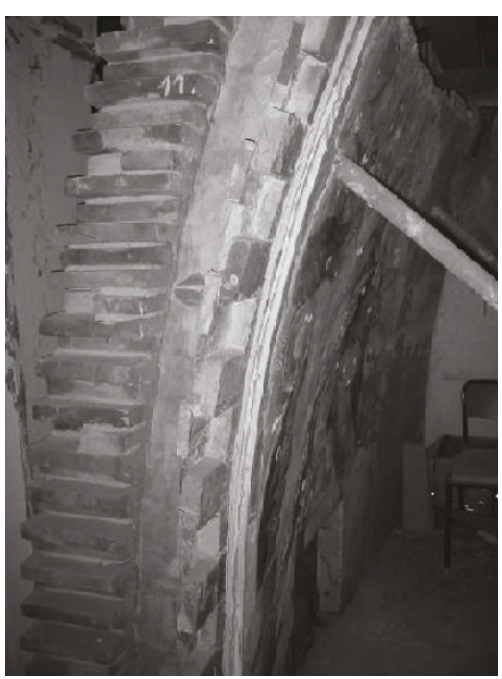

Figure 4: Picture of the $1: 1$ dome scale model.

automatic for every problem. Requirements of both versatility and ease of operation justify not using more advanced systems that could be more adapted to the particular problem considered in this manuscript. Moreover, developing our own signal processing algorithms allows us a total control of the work.

The study was made on a $1: 1$ scale model of the actual dome to overcome the problems of accessibility and the danger of damaging paintings. A photograph of the 1 : 1 scale model is shown in Figure 4. Model dimensions are $2.5 \mathrm{~m}$ width, $2 \mathrm{~m}$ height, and $0.5 \mathrm{~m}$ thick. There is a convex curve in the wall, as in the actual dome.

Relevant information on the acquisition follows:

(i) ultrasonic pulse generation: PR5000 Matec Instruments with a 2500 watts maximum power output;

(ii) transducer: $5.0 \mathrm{MHz} / .250 \mathrm{~KB}-\mathrm{A}$ 66492, Krautkramer, excitation signal $5 \mathrm{MHz}$ burst tone;

(iii) amplifier gain $65 \mathrm{~dB}$;

(iv) analog filter: $2.5 \mathrm{MHz}-6 \mathrm{MHz}$;

(v) tektronix TDS3012 digitalization equipment, sampling frequency $20 \mathrm{MHz}$, amplitude resolution 16 bits, dynamic range $\pm 2.5 \mathrm{~V}$;

(vi) labtop PC for signal transferring and storage.

Note that a $5 \mathrm{MHz}$ burst tone excitation signal was used with the aim of tuning most of the emitted ultrasonic energy in a band centred at $5 \mathrm{MHz}$. Every ultrasonic pulse sent into the material is the result of convolving an (approximate) five cycles segment of a $5 \mathrm{MHz}$ sinusoid with the impulse response of the piezoelectric crystal.

We collected 75 A-scan of 100 microseconds in the locations indicated in Figure 5. The vertical array of locations (separation between two consecutive locations was $2 \mathrm{~cm}$ ) crossed some areas where modifications had been made to the surface (a special type of paper was attached to the wall after preparation of the paintings). This affected the transducer-wall coupling in such a way that different signals were recorded in the affected locations. Note that, except 


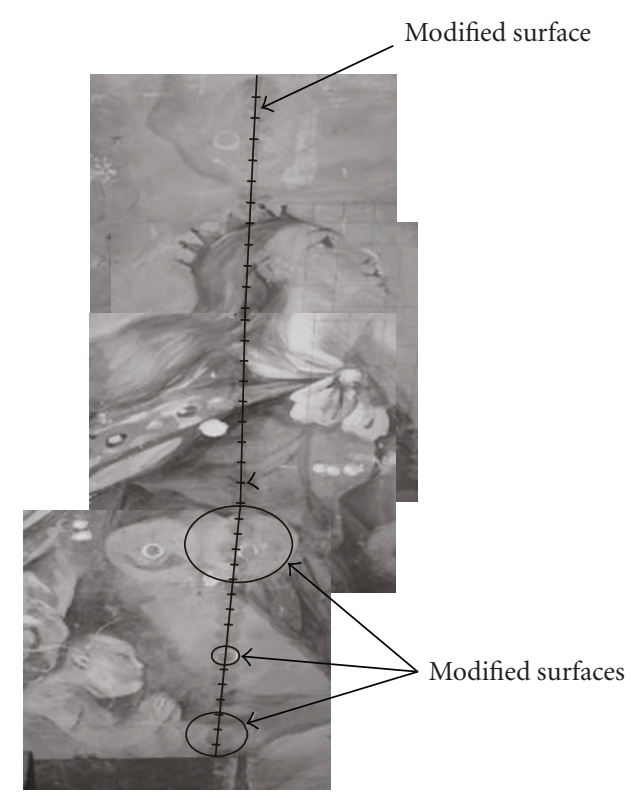

FIgURE 5: Photographic composition indicating the vertical array where the A-scans were collected, and the locations where some modifications were observed in the surface.

for significant changes of the surface, variability of coupling (due, e.g., to different hand pressure on the sensor) may produce variability in the injected ultrasonic energy. But signalto-grain-noise ratio will be the same, so that, in principle, all the four detectors will be affected in a similar manner. The only concern is that ultrasonic energy could reduce in such a manner that reflections from the interface could not be detectable at all.

Normally, the restorer has the prior knowledge about the layer structure of the dome because some destructive inspections have been done in appropriate parts of it, because part of the dome is deteriorated and the inner structure of layers is visible or because there are documents available describing the dome. Thus, the scale model was built after that prior knowledge of the actual dome. The interest for the restorer is to have information about the state of the layers in some specific areas of the domes; in this particular case to know the presence or absence of the mortar layer in every part where painting is to be restored, as explained before. We know that the first layer of mortar (if present) is $0.3 \mathrm{~cm}$ thick, so we can predict what the results should be if the ultrasonic technique could be able to trace the interface between the first and second layers after the first, second, third, ... or $n$th reverberant echoes. We need an estimate of the expected delay between echoes from the interface (the value $T$ in Figure 1). This was done in a small cylindrical section $(0.3 \mathrm{~cm}$ height $)$ of the same type of mortar, by measuring the transmission delay between two identical $5 \mathrm{MHz}$ transducers, each located on the opposite face of the cylinder. A value of 1.92 microseconds was obtained (see Figure 2), so we considered a raw estimate $T=2 \times 1.92=3.84$ microseconds. This meant that a possible first reflection from the interface should arrive at 3.84 microseconds, a second one at
$2 \times 3.84=7.68$ microseconds, a third one at 11.52 microseconds, and so on.

Figure 3 showed the original B-scan (75 A-scans) in the delay interval of 4 to 20 microseconds. This is because the idle time of the receiver is approximately 4 microseconds, and that after 20 microseconds ultrasonic energy practically disappears. This means that the only expected indications (if any) from the interface would be due to a second reflection at a delay of 7.68 microseconds and/or the third reflection at a delay of 11.52 microseconds. This is indicated by two arrows in the axis time of Figure 3. It should be noted that no echo trace from the interface is apparent in the original B-scan, which is composed of multiple echoes, probably coming from surface irregularities and from the mortar grain noise. Note that in the locations corresponding to modified surfaces, the backscattered ultrasonic energy is much lower than in the other locations, hence when we represent all the A-scan together, using a common amplitude scale, it seems that there are no ultrasonic responses at these locations. It should be mentioned that the received signals were prefiltered by an analog bandpass filter adapted to the useful bandwidth $(2.5 \mathrm{MHz}-6 \mathrm{MHz})$, previously to digitalization. However, looking at Figure 3 where we represent the digital records, it can be appreciated that magnitude of grain noise is still comparable to magnitude of the echoes from the interface. That is the essence of the justification for using statistical digital signal processing to extract relevant information. It should be noted that grain noise is due to echoes from small grains of the materials, thus the grain noise power spectral density overlaps with the interface echoes spectrum. That is why the analog pre-filtering does not help us too much in this problem.

Before presenting the results of the processing, we will consider some aspects of the selection of the parameters involved in the algorithms. We need to fit $N$ and $\mathbf{C}_{g}$. The length of the moving window $N$ depends on the duration of the pulse; hence we have estimated "offline" the ultrasonic pulse by using a piece of a material having good ultrasound propagation properties (methacrylate). We measured a duration of the pulse of 1 microseconds (i.e., 5 cycles of the nominal frequency of the $5 \mathrm{MHz}$ transducer). This duration seems to be a correct estimate also for mortar (see the first part of the received signal in Figure 2). In any case, this is not a critical parameter and a raw estimate of the pulse duration suffices. A different matter is the capability for measuring an appropriate waveform for implementing matched filtering; this is the problem with model 1 , as we illustrate with the results below.

On the other hand, we tested different alternatives for estimating the grain noise matrix $\mathbf{C}_{g}$, which produced rather similar results in this application. In the case of the results shown below, they were obtained by estimating a grain noise matrix for every A-scan using the classical sample estimate

$$
\widehat{\mathbf{C}}_{g}=\frac{1}{R} \sum_{i=1}^{R} \mathbf{r}_{i} \mathbf{r}_{i}^{T}
$$

where $\mathbf{r}_{i}, i=1 \cdots R$, indicate all the possible intervals to be processed in the corresponding A-scan. 


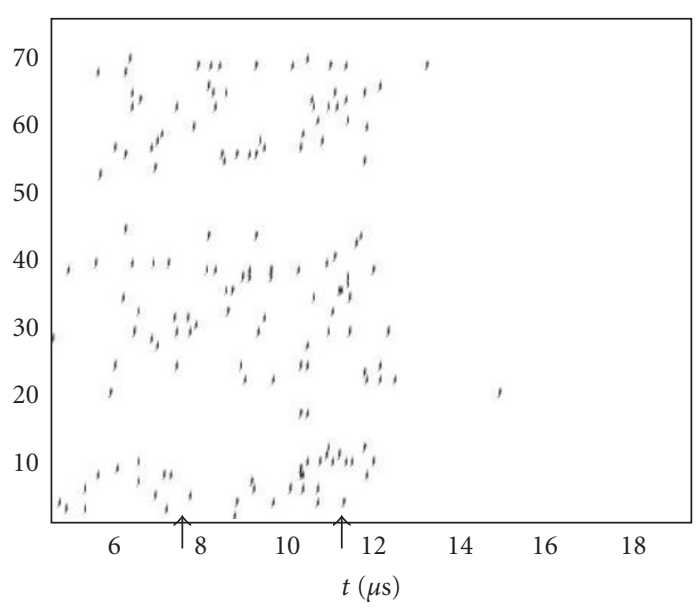

(a)

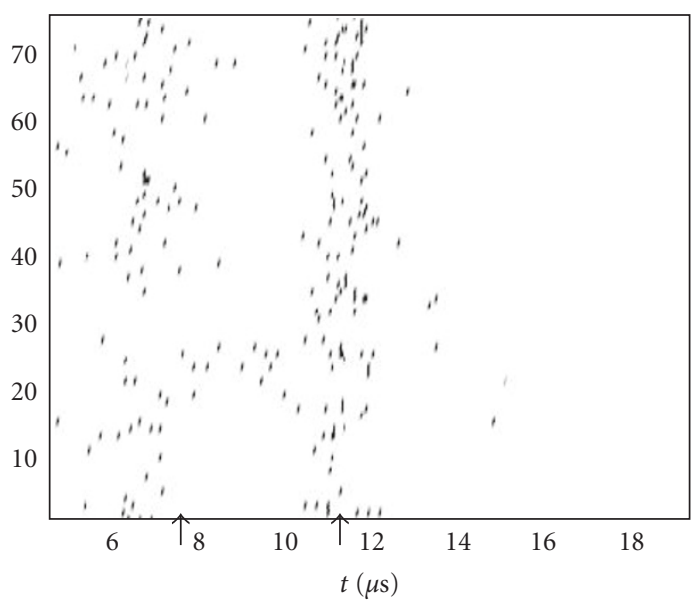

(c)

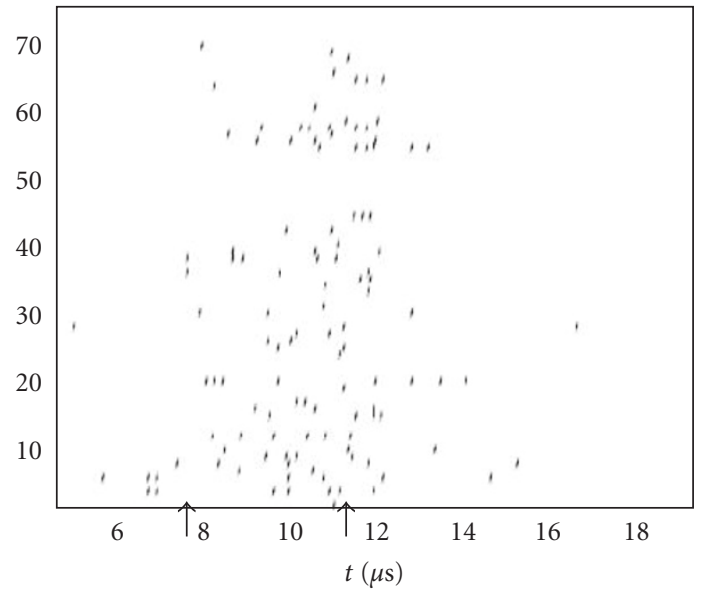

(b)

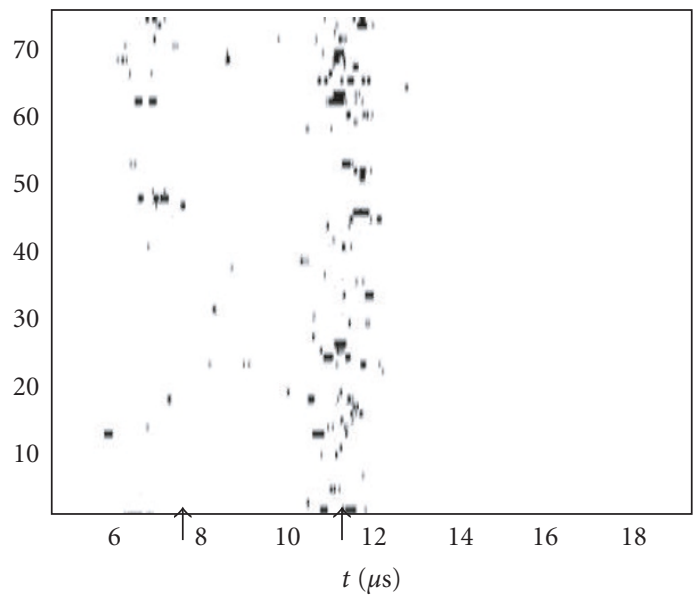

(d)

FIGURE 6: Detection results: (a) matched filter (Model 1), (b) gating of the original signal (Model 2), (c) gating of the prewhitened signal (Model 3), (d) suboptimum technique.

In the Figures 6(a), 6(b), and 6(c), we show the detections (binary B-scan), respectively, obtained with the optimum detectors corresponding to models 1,2 , and 3 . The required vector $\mathbf{s}$ needed in the matched filter detector was obtained from the ultrasonic pulse measured in the methacrylate piece; PFA $=0.001$ in all cases. Detectors corresponding to models 1 and 2 (Figures 6(a) and 6(b)) are not able to obtain the trace of the third reflection. However, the detector of model 3 (Figure 6(c)), which corresponds to a gating of the prewhitened signals, is able to outline the interface. The second reflection is too corrupted by multiple surface and inner reflections to allow reconstruction of the interface trace. A possible fourth reflection seems to be too attenuated to appear. It is noticeable in Figure 6(c) that some detections are also obtained in those scans corresponding to modified surfaces, even though it was no apparent backscattered energy (Figure 3).

For completeness, we have also tried some suboptimum techniques. We use the term suboptimum in the sense that these algorithms do not come from optimum solutions cor- responding to a well-defined model as 1,2 or 3. But they may have general applicability even when the assumptions of models 1, 2, and 3 are not appropriate. For example, Gaussianity is not a correct hypothesis for some coarsegrained materials [5], due to the obtained "spiky" grain noise records, or for materials exhibiting regular spreading of the grains [6]. It is also reasonable to assume that the presence of the interface may alter the grain noise statistics, so that we should consider a different grain noise model under every hypothesis.

These techniques $[2,7]$ decompose the signal into different narrowband frequency channels and nonlinearly process the channel outputs in different forms depending on the particular algorithm selected. Enhancing of the possible presence of the echo is based in the assumption that grain noise will exhibit large level variation at the different channel outputs, meanwhile the possible target echo distributes its energy uniformly among the different channels. In essence, this is a similar assumption to that one made in model 3, because frequency sensitive of the grain noise appears with the linear 
TABLE 1: Quantitative comparison of the results obtained with the different methods (interface vicinity is defined after Figure 6 as the delay interval from 10 to 12.5 microseconds).

\begin{tabular}{l|c|c|c}
\hline & $\begin{array}{l}\text { Mean of number of detections } \\
\text { per scan inside the interface } \\
\text { vicinity }\end{array}$ & $\begin{array}{l}\text { Standard deviation of number of } \\
\text { detections per scan inside the interface } \\
\text { vicinity }\end{array}$ & Mean/Std \\
\hline $\begin{array}{l}\text { Matched filter } \\
\text { (Model 1) }\end{array}$ & 0.84 & 1.15 & 0.730 \\
\hline $\begin{array}{l}\text { Gating of the } \\
\text { original signal } \\
\text { (Model 2) }\end{array}$ & 0.97 & 1.16 & 0.836 \\
\hline $\begin{array}{l}\text { Gating of the } \\
\text { prewhitened signal } \\
\text { (Model 3) }\end{array}$ & 1.52 & 1.29 & 1.178 \\
\hline $\begin{array}{l}\text { Suboptimum } \\
\text { technique }\end{array}$ & 11.15 & 12.98 & 0.859 \\
\hline
\end{tabular}

generative model mentioned above: the echoes due to the grains of the material may add in a constructive (synchronized phase) or destructive manner for every frequency component, thus affecting the grain noise level at every channel output. The difference with model 3 is that now Gaussianity and identical noise distribution under both hypotheses are not assumed. Moreover, there is not any assumption about the pulse waveform $s$ except its insensitivity to the center frequency of the channel.

For a better comparison we have also adopted a detection approach to the suboptimum algorithms. First, we compute the discrete Fourier transform (DFT) of every vector $\mathbf{r}_{w}=\mathbf{C}_{g}^{-1 / 2} \mathbf{r}$. This is a simple form of implementing the frequency channels. On the other hand, prewhitening is required to equalize the pulse spectrum. Second, a given band centred at the transducer nominal frequency is selected and a detector is applied to every frequency bin inside the selected band. Finally, hypothesis $H_{1}$ (presence of interface echo) is accepted when all the individual detectors are in favor of $H_{1}$. The corresponding algebraic expression of the algorithm, preserving as much as possible the notation used until now, is given by

$$
\begin{aligned}
& z_{i}\left(n_{s}\right)=f_{i}(\mathbf{r})=\mathbf{r}_{w}^{T} \mathbf{e}_{i}, \\
& \mathbf{e}_{i}=\left[1 e^{-j(2 \pi / N) i} e^{-j(2 \pi / N) i \cdot 2} \cdots e^{-j(2 \pi / N) i \cdot(N-1)}\right]^{T}, \\
& 0<i_{l} \leq i \leq i_{u}<N-1 ;
\end{aligned}
$$

if $\left|z_{i}\left(n_{s}\right)\right|>t$ for all $i, \quad$ decide $H_{1}$, otherwise, decide $H_{2}$.

Note that $\mathbf{e}_{i}$ is the DFT vector tuned to normalized frequency $i / N$ and that $i_{l}$ and $i_{u}$ are respectively the lower and upper bins delimiting the band of analysis. This latter must coincide with the band of the pulse, so that actually some knowledge about $\mathbf{s}$ is also required.

We have tested the detector of (12) in the dome application. The band of analysis has been determined from the same "offline" pulse estimate used in the matched filter and it coincides with the useful bandwidth established by the ana- $\log$ filter $(2.5-6 \mathrm{MHz})$. The sampling frequency was $20 \mathrm{MHz}$. This implies a useful normalized bandwidth of $(6-2.5) / 20=$ 0.175 . On the other hand, the pulse duration was 1 microseconds, that is, 20 samples at a sampling frequency of $20 \mathrm{MHz}$, hence we fitted $N=20$. Therefore, in the normalized interval of 0.175 , we have $20 \times 0.175=3.5$ independent bins available for implementing (12). Actually we used bins 3, 4, and 5 corresponding respectively to the analog frequencies 3, 4 and $5 \mathrm{MHz}$. Results are shown in Figure 6(d). It can be seen that there are no significant improvements with respect to a gating of the prewhitened signal. Although, in general, the suboptimum technique produces a "cleaner" B-scan, the trace of the third reflection is worse outlined. With respect to the second reflection, we see again that it is not detected at all. As we already mentioned, it seems to be too corrupted by multiple surface and inner reflections to allow reconstruction of the interface trace. Note that surface reflections are produced only once, but interface reflections have a reverberation effect and (except for the progressive echo attenuation) could appear several times along the ultrasonic records. On the other hand, attenuation model of grain noise could be different from the attenuation model of interface echoes, thus justifying the possibility of detecting the third reflection but not the second one, because this later could be more corrupted by grain noise, in spite of its larger amplitude.

We have also computed some values from Figure 6 with the aim of having some quantitative comparison among the different methods. These values should be considered complementary information to the (qualitative) direct observation of Figure 6 . The computed values are indicated in Table 1. For every method we had counted the number of detections inside the vicinity of the interface (defined after Figure 6, as the delay interval from 10 to 12.5 microseconds). Then, we have computed the mean number of detections per A-scan inside the interface vicinity (i.e., total number of detections inside the vicinity divided by 75 , the total number of A-scans) and the corresponding standard deviation. This latter value gives us some insight into the degree of uniformity in the distribution of detections among the different scans. 


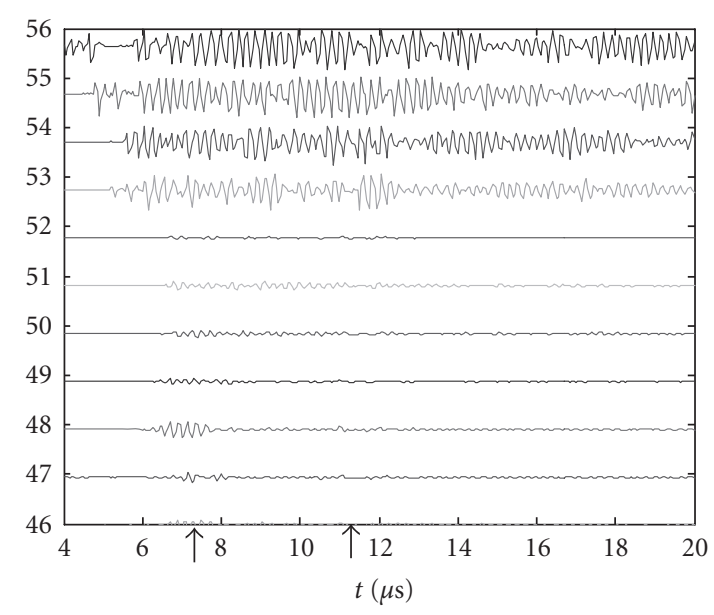

(a)

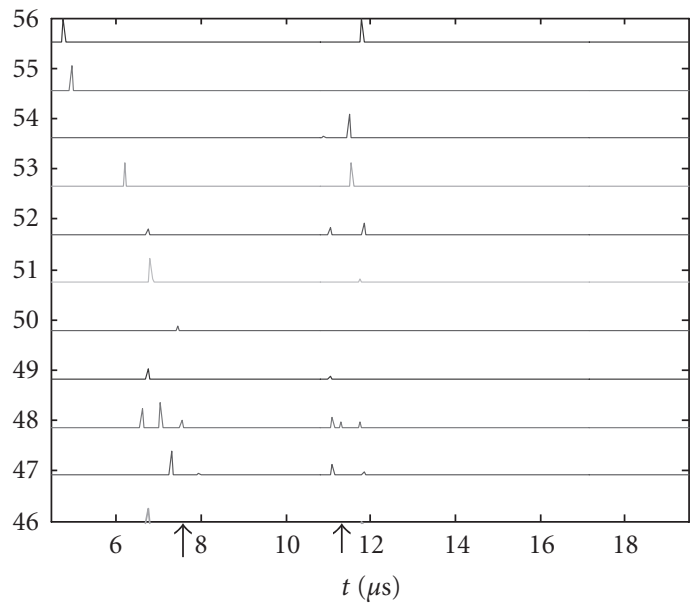

(c)

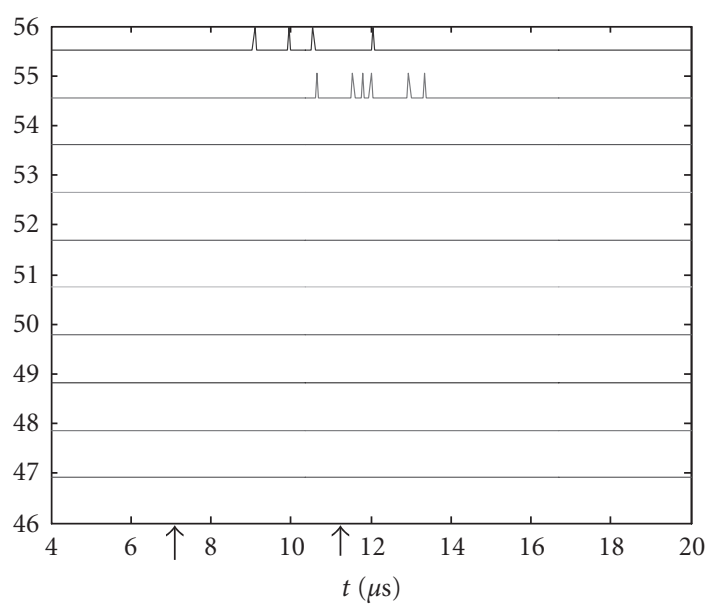

(b)

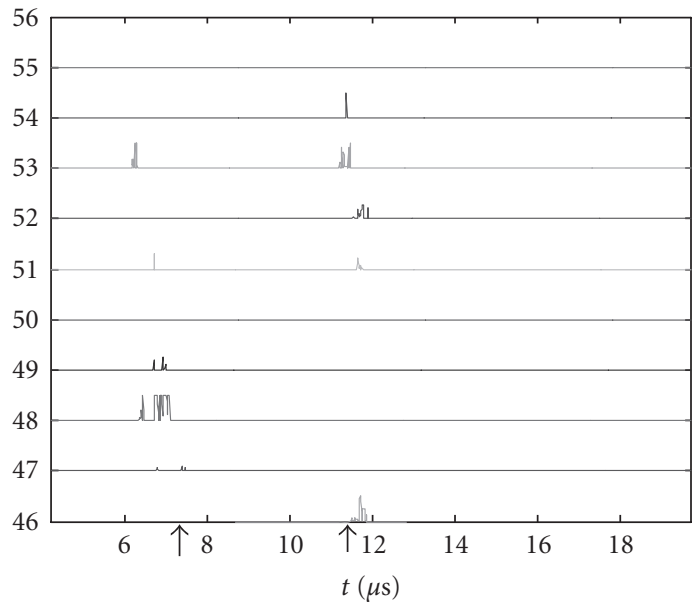

(d)

Figure 7: Processed A-scans (46 to 56): (a) original A-scans, (b) matched filter (Model 1), (c) gating of the prewhitened signal, (Model 3 ), (d) suboptimum technique.

Note that, in principle, we should receive echoes from the interface in all the scans, as we know a priori that the mortar layer is always present in the scale model of the dome. In consequence, the normalized mean (last column in Table 1) may be a valuable figure of merit to evaluate the quality of the corresponding method in conjunction with the qualitative information. Model 3 gives the largest normalized mean. The suboptimum technique gives significantly more detections per scan than the optimum techniques, but variance is very large (see in Figure 6(d) that there are a lot of detections in some scans but only a little or even zero in many other).

To gain further insights into the capability of the methods to deal with the grain noise problem, we have represented in Figure 7 the processed A-scans. This has been done after (4), that is, every time a detection is produced, we keep the (magnitude) of the sample value, otherwise a zero is given. We have selected in Figure 7 the scans 46 to 56, which includes the modified surface section where, apparently, there was no ultrasonic energy. Note that only model 3 and suboptimum techniques exhibit a significant signal level at the delays corresponding to the third echo, including some of the A-scans corresponding to the modified surface.

We conclude that in this application, the hypothesis assumed in model 3 seems to be appropriate for a reasonable extraction of the interface trace.

\section{CONCLUSIONS}

We have presented in this paper the application of optimum detectors to the problem of outlining the interface between the first and second layer of a dome. From a signal processing perspective, the problem is automatic detection of pulses embedded in a grain noise background. We have considered three models and their corresponding solutions: matched filter, gating of the original signal, and gating of the prewhitened original signal. The use of a matched filter requires knowledge of the waveform of the signal which is to be detected. Gating of the original signal is optimum only 
if the pulse verifies a condition which cannot be justified by physical arguments of grain noise generation. However, gating of the prewhitened original signal is optimum if the grain noise admits a linear generative model consisting in the convolution of the material reflectivity and the pulse waveform. A suboptimum technique exploiting frequency sensitivity of grain noise has also been tested with no significant improvements with respect to the prewhitening of the original signal. Therefore, model 3 seems to be appropriate in the considered application.

Although focused to dome analysis, the general procedure followed in this work may be applied in other nondestructive analysis involving materials which produce high levels of grain noise.

\section{APPENDIX}

Let us express the hypotheses of (1) in vector form (to ease the notation dependence on $n_{s}$ of the different vectors is not expressed)

$$
\begin{array}{lll}
H_{1} & \mathbf{r}=\mathbf{p}+\mathbf{g}, & \mathbf{p}=\left[p\left(n_{s}\right) \cdots p\left(n_{s}+N-1\right)\right]^{T}, \\
H_{2} & \mathbf{r}=\mathbf{g}, & \mathbf{g}=\left[g\left(n_{s}\right) \cdots g\left(n_{s}+N-1\right)\right]^{T} .
\end{array}
$$

The optimum detector is obtained by comparing the loglikelihood ratio with a threshold $\lambda$ [3]. The log-likelihood ratio is the quotient of the probability density functions of the observation vector $\mathbf{r}$ conditioned to hypotheses $H_{1}$ and $\mathrm{H}_{2}$, respectively, that is,

$$
\log \frac{P\left(\mathbf{r} / H_{1}\right)}{P\left(\mathbf{r} / H_{2}\right)} \underset{H_{2}}{\stackrel{H_{1}}{\gtrless}} \lambda
$$

Given the conditions of Model 1, we have that both $P\left(\mathbf{r} / H_{1}\right)$ and $P\left(\mathbf{r} / H_{2}\right)$ will be multivariate Gaussian having vector mean $\mathbf{0}$ and $a \mathbf{s}$, respectively

$$
\begin{aligned}
& P\left(\mathbf{r} / H_{1}\right)=\frac{1}{\sqrt{(2 \pi)^{N}\left|\mathbf{C}_{g}\right|}} \exp \left\{-\frac{1}{2}(\mathbf{r}-a \mathbf{s})^{T} \mathbf{C}_{g}^{-1}(\mathbf{r}-a \mathbf{s})\right\}, \\
& P\left(\mathbf{r} / H_{2}\right)=\frac{1}{\sqrt{(2 \pi)^{N}\left|\mathbf{C}_{g}\right|}} \exp \left\{-\frac{1}{2} \mathbf{r}^{T} \mathbf{C}_{g}^{-1} \mathbf{r}\right\} .
\end{aligned}
$$

Substituting in (A.2), we arrive to

$$
a \mathbf{r}^{T} \mathbf{C}_{g}^{-1} \mathbf{s}-a^{2} \mathbf{s}^{T} \mathbf{C}_{g}^{-1} \mathbf{s} \underset{H_{2}}{\stackrel{H_{1}}{\gtrless}} \lambda \Longleftrightarrow \mathbf{r}^{T} \mathbf{C}_{g}^{-1} \mathbf{s} \underset{H_{2}}{\stackrel{H_{1}}{\gtrless}} \frac{\lambda}{a}+a \mathbf{s}^{T} \mathbf{C}_{g}^{-1} \mathbf{s}=\lambda^{\prime} .
$$

Under $H_{2}$, the statistic $z\left(n_{s}\right)=\mathbf{r}^{T} \mathbf{C}_{g}^{-1} \mathbf{s}$ is a zero mean Gaussian random variable having unit variance so that $\lambda^{\prime}$ can be easily computed to obtain a given PFA. Optimality guarantees that PD will be maximum.

\section{ACKNOWLEDGMENTS}

This work has been supported by Spanish Administration, under Grant TEC2005-01820, and by European Community, FEDER program.

\section{REFERENCES}

[1] J. Gosálbez, A. Salazar, I. Bosch, R. Miralles, and L. Vergara, "Application of ultrasonic nondestructive testing to the diagnosis of consolidation of a restored dome," Materials Evaluation, vol. 64, no. 5, pp. 492-497, 2006.

[2] M. G. Gustafsson, "Nonlinear clutter suppression using split spectrum processing and optimal detection," IEEE Transactions on Ultrasonics, Ferroelectrics, and Frequency Control, vol. 43, no. 1, pp. 109-124, 1996.

[3] L. L. Scharf, Statistical Signal Processing, Addison-Wesley, Reading, Mass, USA, 1991.

[4] L. Vergara, J. Gosálbez, J. V. Fuente, R. Miralles, and I. Bosch, "Measurement of cement porosity by centroid frequency profiles of ultrasonic grain noise," Signal Processing, vol. 84, no. 12, pp. 2315-2324, 2004.

[5] L. Vergara and J. M. Páez, "Backscattering grain noise modelling in ultrasonic non-destructive testing," Waves in Random Media, vol. 1, no. 1, pp. 81-92, 1991.

[6] V. M. Narayanan, R. C. Molthen, P. M. Shankar, L. Vergara, and J. M. Reid, "Studies on ultrasonic scattering from quasiperiodic structures," IEEE Transactions on Ultrasonics, Ferroelectrics, and Frequency Control, vol. 44, no. 1, pp. 114-124, 1997.

[7] L. Ericsson and T. Stepinski, "Algorithms for suppressing ultrasonic backscattering from material structure," Ultrasonics, vol. 40, no. 1-8, pp. 733-734, 2002.

Luis Vergara was born in Madrid (Spain) in 1956. He received the Ingeniero de Telecomunicación and the Doctor Ingeniero de Telecomunicación degrees from the Universidad Politécnica de Madrid (UPM) in 1980 and 1983, respectively. Until 1992, he worked at the Departamento de Señales, Sistemas y Radiocomunicaciones (UPM) as an Associate Professor. In 1992 he joined the Departamento de Comunicaciones, Universidad Politécnica de Valencia (UPV), Spain, where he became Professor and where he was Department Head until April 2004. From April 2004 to April 2005 he was Vicerector of New Technologies at the UPV. He is now responsible for the Signal Processing Group of the UPV, a member group of the Institute of Telecommunication and Multimedia Applications (I-TEAM) of UPV. His research concentrates in the statistical signal processing area, where he has worked in different theoretical and applied problems, many of them under contract with the industry. His theoretical aspects of interest are signal detection and classification, independent component analysis, and spectral analysis. Currently, he is involved in ultrasound signal processing for nondestructive evaluation, in infrared signal processing for fire detection and in cognitive audio for surveillance applications. He has published more than 150 papers including journals and conference contributions. 
Ignacio Bosch was born in Valencia (Spain) in 1975 . He received the Ingeniero de Telecomunicación and the Doctor Ingeniero de Telecomunicación degrees from the Universidad Politécnica de Valencia (UPV) in 2001 and 2005, respectively. $\mathrm{He}$ is an Assistant Professor at Departamento de Comunicaciones, UPV and member of the Signal Processing Group of the Institute of Telecommunication and Multimedia Applications

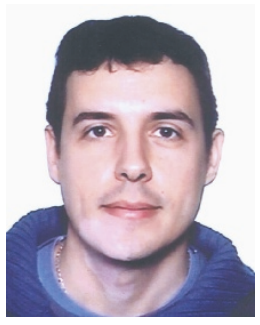
(I-TEAM) of UPV. His research concentrates in the statistical signal processing area, where he has worked in different theoretical and applied problems, many of them under contract with the industry. His theoretical aspects of interest are signal detection and classification and decision fusion. Currently, he is involved in infrared signal processing for early warning of forest fires. He has published more than 40 papers including journals and conference contributions.

Jorge Gosálbez was born in Valencia (Spain) in 1975. He received the Ingeniero de Telecomunicación and the Doctor Ingeniero de Telecomunicación degrees from the Universidad Politécnica de Valencia (UPV) in 2000 and 2004, respectively. $\mathrm{He}$ is an Assistant Professor at Departamento de Comunicaciones UPV and member of the Signal Processing Group of the Institute of Telecommunication and Multi-

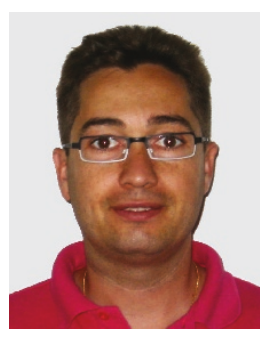
media Applications (I-TEAM) of UPV. His research concentrates in the statistical signal processing area, where he has worked in different theoretical and applied problems, many of them under contract with the industry. His theoretical aspects of interest are timefrequency analysis, signal detection, and array processing. Currently, he is involved in ultrasound signal processing for nondestructive evaluation of materials, in surveillance systems based on acoustic information and in acoustic source location and tracking based on sensor and array signal processing. He has published more than 50 papers including journals and conference contributions.

Addisson Salazar is working towards the Doctorate degree in Telecommunications at Universidad Politécnica de Valencia (UPV). $\mathrm{He}$ has received the B.S. and M.S. degrees in Informatics from Universidad Industrial de Santander and the D.E.A. degree in Telecommunications from UPV in 2003. $\mathrm{He}$ is a researcher of the Signal Processing Group of the Institute of Telecommunication and Multimedia Applications at UPV.

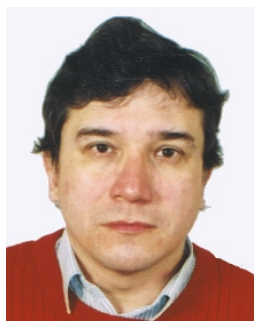

His research interest is focused on statistical signal processing, pattern recognition, data mining, and knowledge discovery, where he has worked in different theoretical and applied problems, many of them under contract with the industry. His theoretical aspects of interest are signal classification, time-frequency analysis, independent component analysis, and algorithms for data mining. He has published more than 70 papers including journals and conference contributions. 